\title{
PERFECT RECONSTRUCTION FILTER BANKS FOR HDTV REPRESENTATION AND CODING*
}

\author{
Martin VETTERLI, Jelena KOVAČEVIĆ † \\ Department of Electrical Engineering and Center for Telecommunications Research, Columbia University, New York, NY \\ 10027-6699, U.S.A.
}

Didier J. LEGALL

C-Cube Microsystems, 399-A West Trimble Road, San Jose, CA 95131, U.S.A.

Received 1 September 1989

Revised 7 May 1990

\begin{abstract}
Subband decomposition of HDTV signals is important both for representation purposes (to create compatible subchannels) and for coding (several proposed compression schemes include some subband division). We first review perfect reconstruction filter banks in multiple dimensions in the context of arbitrary sampling patterns. Then we concentrate on the special case of quincunx subsampling and derive filter banks to go from progressive to interlaced scanning (with a highpass which contains deinterlacing information) as well as from interlaced to progressive. We apply this decomposition to a sequence and indicate bitrates.
\end{abstract}

Keywords. HDTV, subband decomposition, filter banks.

\section{Introduction}

Multirate techniques and filter banks have emerged as useful concepts in image and video processing. They can be used to create signal hierarchies where subchannels are compatible with existing standards [12]. They are central to subband coding techniques which have been successfully used in HDTV compression [3,11]. So far, subband decomposition has been applied mostly in a separable fashion both in two [24] and three dimensions [9]. Non-separable schemes that have been used include quincunx intraframe subsampling $[1,3]$ and hexagonal subsampling $[1,13]$. Obviously, in

* This is an extended version of a paper presented at the Third International Workshop on HDTV in Torino, September 1989.

+ Work supported in part by the National Science Foundation under grants CDR-84-21402 and MIP-88-08277. three dimensions, there are many possible subsampling schemes [5] and associated filter banks. One of immediate interest for television is quincunx subsampling over the [time, vertical]-plane, since it allows to go from progressive to interlaced scanning, as well as from interlaced to progressive [20].

In the first case, a progressive sequence is filtered with a diamond shaped lowpass filter and then quincunx subsampled to yield an interlaced sequence. A complementary highpass version, also quincunx subsampled, is the deinterlacing channel (also called a 'helper' signal [15]), since when combined with the lowpass version and using appropriate filtering, it allows perfect recovery of the original progressive sequence. Figure 1 depicts this process schematically. Earlier proposals in this direction have been made in $[7,15]$ but do not achieve perfect reconstruction. Note that this is 
a)

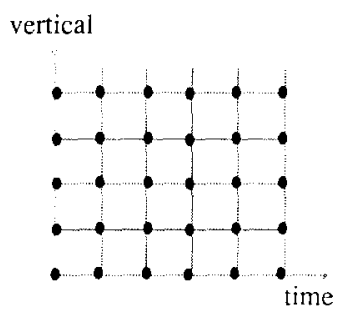

vertical

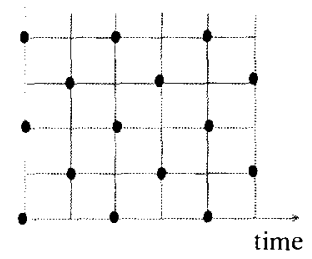

b)

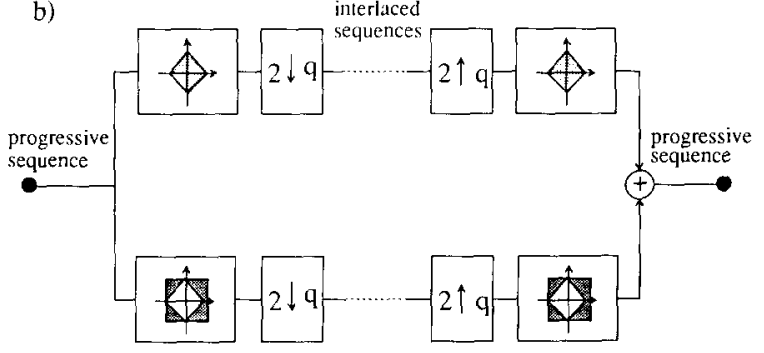

Fig. 1. Progressive to interlaced conversion: (a) rectangular and quincunx lattices, (b) filter bank decomposition of one progressive into two interlaced sequences.

a)

vertical

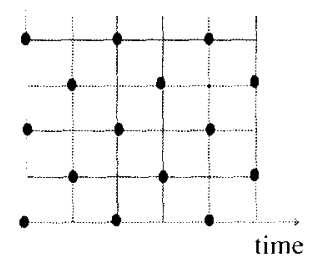

vertical

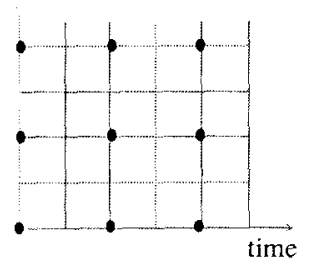

b)

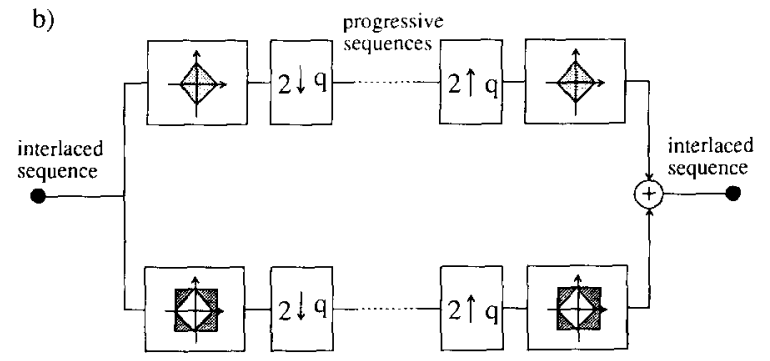

Fig. 2. Interlaced to progressive conversion: (a) quincunx and rectangular lattices, (b) filter bank decomposition of one interlaced into two progressive sequences.

quite different from standard deinterlacing procedures, which do not require an auxiliary 'deinterlacing' channel but cannot guarantee a perfect recovery of the progressive sequence. Note also that the above approaches are nonlinear [23].

In the second case, an interlaced sequence is separated into lowpass and highpass versions, again with diamond shaped filters, and then quincunx subsampled. This produces two progressive sequences, from which the original interlaced sequence can be perfectly recovered. A schematic representation is given in Fig. 2. Such a scheme would be useful for coding purposes, since the highpass version, due to its low energy content, will be relatively easy to code, and the lowpass version is well suited for motion based processing because of its progressive nature.

A possible combination of the two steps above is shown in Fig. 3. The initial progressive sequence is split into a deinterlacing part as well as a lowpass interlaced sequence. This latter sequence is then further split into two progressive sequences.

The design of filters for perfect reconstruction banks has been studied extensively, and results on filter structures are available in the onedimensional case $[16,17,21]$. In two or more dimensions, results on aliasing cancellation and perfect reconstruction have been derived, and

a)
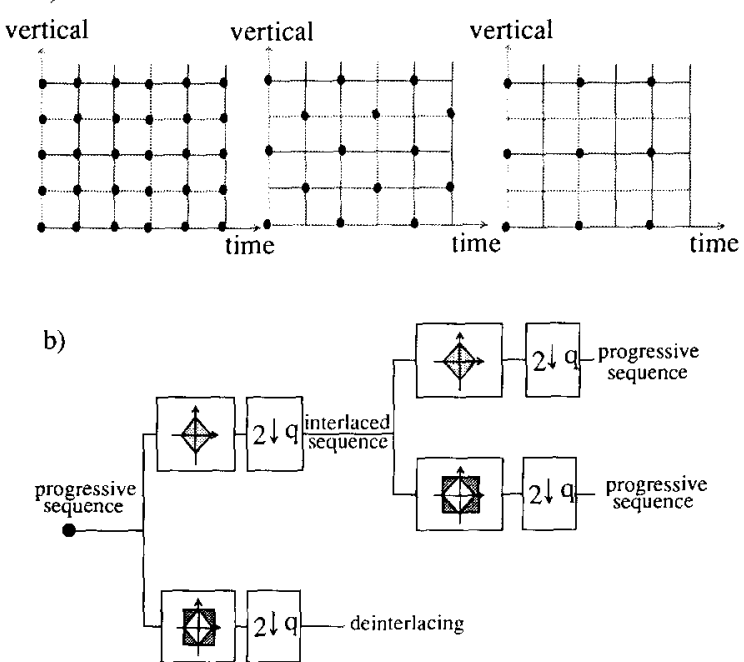

Fig. 3. Two step quincunx subsampling of a progressive sequence resulting into one interlaced sequence and two progressive sequences: (a) the sampling lattices involved, (b) filter bank decomposition. 
some initial designs are available $[1,8,22]$. However, more effort is required to design practical filters for specific applications and, on the theoretical side, issues like completeness of filter structures are quite delicate. In this paper we will concentrate on structures for the particular case of quincunx subsampling, since this is of most practical interest. A solution using IIR filters was derived in [2] but leads to non-causal synthesis. We will concentrate on FIR solutions, because our filters are used over time and causality is thus necessary. A para-unitary FIR solution appears in [22], and we derive below a linear phase solution as well as structures for both para-unitary and linear phase filters allowing perfect reconstruction in the quincunx subsampling case. The structures lead to very low complexity filter banks.

The outline of the paper is as follows. Section 2 reviews perfect reconstruction filter banks with aribtrary sampling patterns and indicates results on alias-free and perfect reconstruction. Section 3 specializes these results to the quincunx subsampling case which is of particular interest for video processing. Section 4 derives cascade structures for perfect reconstruction filter banks. Section 5 shows the application to video signals, with progressive to interlaced as well as interlaced to pro- gressive channels. Then, Section 6 shows the results obtained on a sequence, and indicates bit-rates of the highpass channel after run-length encoding. Finally, Section 7 discusses the results and indicates further work, while Appendices A-C discuss some structural properties of the filters for the quincunx sampling case. Let us also point out that in what follows 'reversal' will entail reversing the directions of the axes in question (for example reversing the direction of the vertical and time axes in our specific application).

\section{Perfect reconstruction filter banks with arbitrary sampling patterns}

We will keep our discussion to the twodimensional case, but most results hold for an arbitrary number of dimensions.

Consider an analysis/synthesis filter bank as shown in Fig. 4. As can be seen from the figure, the two basic operations performed are filtering and sampling. The sampling process in multiple dimensions can be represented by a lattice, which is defined as the set of all linear combinations of two basis vectors $\boldsymbol{a}_{1}$ and $\boldsymbol{a}_{2}$ with integer coefficients $[4,5]$. Thus a point on the input lattice $\left(n_{1}, n_{2}\right)$ is

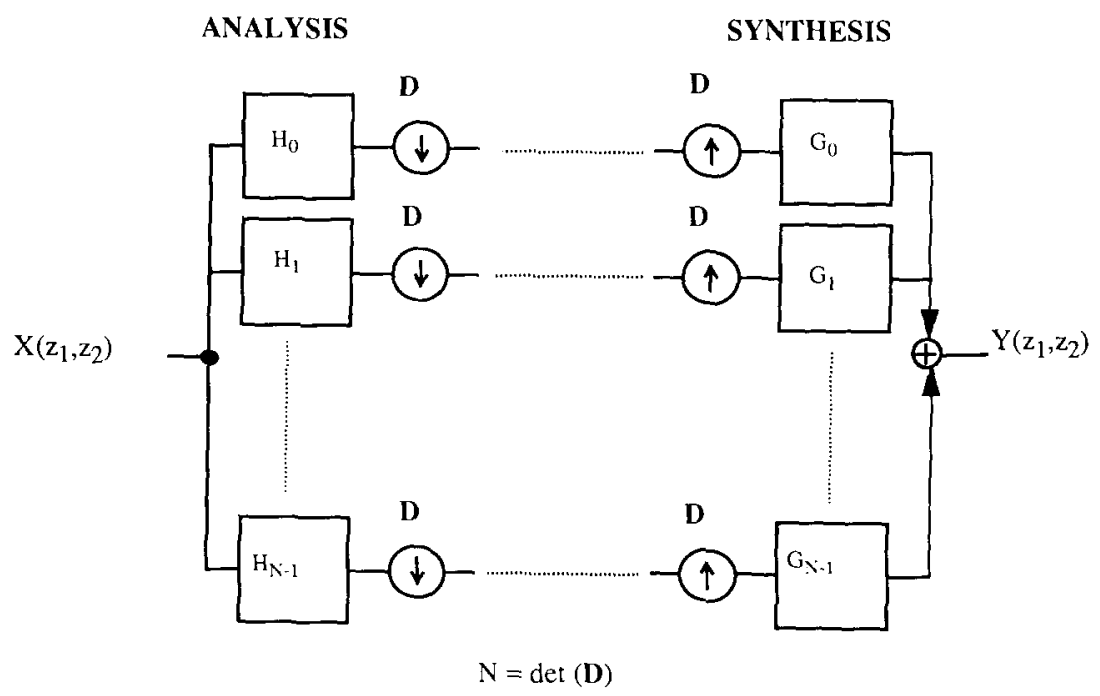

Fig. 4. An analysis/synthesis two-dimensional filter bank. The sampling pattern used is characterized by the sampling matrix $D$. 
related to the one on the output lattice $\left(u_{1}, u_{2}\right)$ by

$$
\left[\begin{array}{l}
n_{1} \\
n_{2}
\end{array}\right]=\left[\begin{array}{ll}
d_{00} & d_{01} \\
d_{10} & d_{11}
\end{array}\right]\left[\begin{array}{l}
u_{1} \\
u_{2}
\end{array}\right]=D \cdot u
$$

where $D$ is the matrix characterizing the sampling process. Note that $\boldsymbol{D}$ is not unique for a given sampling pattern and that two matrices representing the same sampling process are related by a linear transformation represented by an integer matrix with determinant equal to one [4]. Note also that one can always choose $d_{10}=0, d_{00}>0$ and $0 \leqslant d_{01}<d_{00}$. The number of input lattice samples contained in the fundamental parallelepiped (i.e., the parallelepiped formed by the two basis vectors) represents the reciprocal of the sampling density and is given by $N=\operatorname{det}[D]=$ $d_{00} d_{11}-d_{01} d_{10}$. Shifting the origin of the output

a)
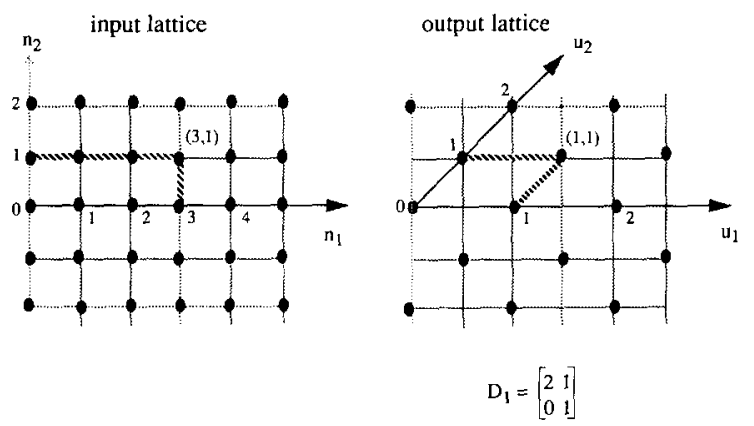

b)
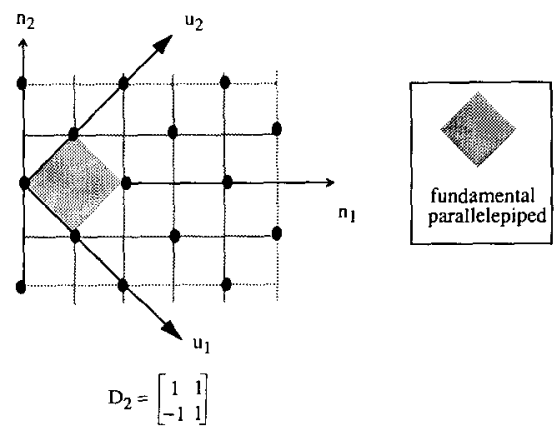

Fig. 5. Quincunx sampling lattice. (a) Conversion from the input lattice to the ouput (quincunx) lattice with matrix $D_{1}$. The point $(1,1)$ on the quincunx lattice corresponds to the point $(3,1)$ on the input lattice. (b) Conversion using the matrix $\mathrm{D}_{2}$. lattice to any of the points of the input lattice yields a so-called coset. Clearly there are exactly $N$ distinct cosets obtained by shifting the origin of the output lattice to the points of the fundamental parallelepiped. The union of all cosets for a given lattice yields the input lattice. Figure 5 depicts the sampling process schematically in the quincunx case with two possible sampling matrices:

$$
D_{1}=\left[\begin{array}{ll}
2 & 1 \\
0 & 1
\end{array}\right], \quad D_{2}=\left[\begin{array}{rr}
1 & 1 \\
-1 & 1
\end{array}\right]
$$

The above matrices represent obviously the same sampling process and thus they are related by a matrix with determinant equal to one:

$$
\begin{aligned}
\boldsymbol{D}_{1} & =\left[\begin{array}{ll}
2 & 1 \\
0 & 1
\end{array}\right]=\left[\begin{array}{rr}
1 & 1 \\
-1 & 1
\end{array}\right]\left[\begin{array}{ll}
1 & 0 \\
1 & 1
\end{array}\right] \\
& =\boldsymbol{D}_{2}\left[\begin{array}{ll}
1 & 0 \\
1 & 1
\end{array}\right] .
\end{aligned}
$$

Consider for example sampling with matrix $\boldsymbol{D}_{\mathbf{1}}$ (Fig. 5(a)). Then the point $\left(u_{1}, u_{2}\right)=(1,1)$ on the output lattice corresponds to

$$
\left[\begin{array}{l}
n_{1} \\
n_{2}
\end{array}\right]=\left[\begin{array}{ll}
2 & 1 \\
0 & 1
\end{array}\right]\left[\begin{array}{l}
1 \\
1
\end{array}\right]=\left[\begin{array}{l}
3 \\
1
\end{array}\right]
$$

on the input lattice. Figure 6 shows the lattice together with its cosets in the hexagonal case. The subsampling operation is periodically shift variant, since samples of the input at locations $\boldsymbol{D} \cdot \boldsymbol{u}$ are kept while all others are dropped. It is this shift variance that leads to aliased versions of the input signal in the output. In a subband coding system,

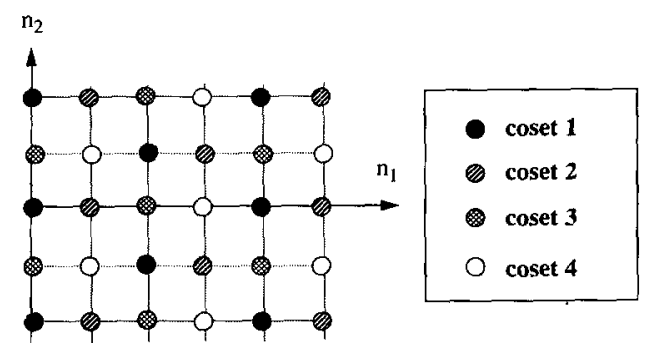

Fig. 6. The four cosets corresponding to the hexagonal sampling structure. Their union yields the original lattice. 
dropped samples will be replaced by zeroes (corresponding to an upsampling by $N$ ) before entering the synthesis bank, and thus, the down-andupsampling process is equivalent to a modulation by a function $f\left(n_{1}, n_{2}\right)$ which equals 1 at locations $\boldsymbol{D} \cdot \boldsymbol{u}$ and zero elsewhere:

$$
f\left(n_{1}, n_{2}\right)=\frac{1}{N} \sum_{k=0}^{d_{00}-1} \sum_{l=0}^{d_{11}-1} W_{N}^{d_{11} n_{1} k+d_{00} n_{2} l-d_{01} n_{2} k}
$$

where $W_{N}$ is the $N$ th root of unity and the upper triangular form of the sampling matrix was used (the one where $d_{10}=0$ ). By the modulation theorem, this clearly produces $N-1$ aliased versions for $k$ or $l \neq 0$. A convenient way to take care of the shift variance of such a multidimensional multirate system is to decompose both signals and filters into so-called polyphase components, each one corresponding to one of the cosets of the output lattice. For example, the filter coefficients at the points of one of the cosets represent actually the response of the filter to the impulse at the location which is a space reversed image of the point in the fundamental parallelepiped corresponding to that coset. In this polyphase domain, the system becomes shift invariant. Thus, signals at the output of the analysis bank can be represented in terms of the input signal, forward polyphase transform and the analysis polyphase matrix $\boldsymbol{H}_{\mathrm{p}}\left(z_{1}, z_{2}\right)$ (that is, the matrix containing polyphase components of the analysis filters), while the output signal can be represented in terms of the input channel signals, the synthesis polyphase matrix $\boldsymbol{G}_{\mathrm{p}}\left(z_{1}, z_{2}\right)$ (that is, the matrix containing polyphase components of the synthesis filters) and the inverse polyphase transform. For more details on polyphase matrices see [19].

Conditions for aliasing cancellation are given in [8] and are expressed in terms of the transfer function matrix $\boldsymbol{T}_{\mathrm{p}}\left(z_{1}, z_{2}\right)$ obtained as the product of the polyphase matrices of the synthesis and analysis bank:

$$
\boldsymbol{T}_{\mathrm{p}}\left(z_{1}, z_{2}\right)=\boldsymbol{G}_{\mathrm{p}}\left(z_{1}, z_{2}\right) \cdot \boldsymbol{H}_{\mathrm{p}}\left(z_{1}, z_{2}\right) .
$$

Perfect reconstruction (i.e., the output signal is a delayed and possibly scaled version of the input signal) is achieved typically when $T_{\mathrm{p}}\left(z_{1}, z_{2}\right)=I$ or a shifted version thereof. Thus, the filter design problem we are faced with is to find a useful set of filters so that $\boldsymbol{T}_{\mathrm{p}}\left(z_{1}, z_{2}\right)$ corresponds to perfect reconstruction. Several approaches are possible depending on what constraints have to be met. For example, one can require the filter bank to be para-unitary [16], that is,

$$
\boldsymbol{H}_{\mathrm{p}}^{\mathrm{T}}\left(z_{1}^{-1}, z_{2}^{-1}\right) \cdot \boldsymbol{H}_{\mathrm{p}}\left(z_{1}, z_{2}\right)=\boldsymbol{I},
$$

or one can impose a linear phase on all filters.

\section{Perfect reconstruction filter banks for quincunx sampling}

Let us look specifically at the quincunx subsampling case. The output of an analysis/synthesis system with quincunx subsampling, as depicted in Fig. 1 , is given by [18]

$$
\begin{aligned}
\hat{X}\left(z_{1}, z_{2}\right)=\frac{1}{2}\left[G_{0}\left(z_{1}, z_{2}\right)\right. & \left.G_{1}\left(z_{1}, z_{2}\right)\right] \\
. & {\left[\begin{array}{cc}
H_{0}\left(z_{1}, z_{2}\right) & H_{0}\left(-z_{1},-z_{2}\right) \\
H_{1}\left(z_{1}, z_{2}\right) & H_{1}\left(-z_{1},-z_{2}\right)
\end{array}\right] } \\
& \cdot\left[\begin{array}{c}
X\left(z_{1}, z_{2}\right) \\
X\left(-z_{1},-z_{2}\right)
\end{array}\right] .
\end{aligned}
$$

To cancel the aliased version of the input signal $X\left(-z_{1},-z_{2}\right)$ in the output we can choose the synthesis filters as follows:

$$
\begin{aligned}
& G_{0}\left(z_{1}, z_{2}\right)=H_{1}\left(-z_{1},-z_{2}\right), \\
& G_{1}\left(z_{1}, z_{2}\right)=-H_{0}\left(-z_{1},-z_{2}\right) .
\end{aligned}
$$

Substituting (9) into (8) we obtain

$$
\begin{aligned}
\hat{X}\left(z_{1}, z_{2}\right)= & \frac{1}{2}\left(H_{1}\left(-z_{1},-z_{2}\right) H_{0}\left(z_{1}, z_{2}\right)\right. \\
& \left.-H_{0}\left(-z_{1},-z_{2}\right) H_{1}\left(z_{1}, z_{2}\right)\right) \\
& \cdot X\left(z_{1}, z_{2}\right),
\end{aligned}
$$

which depends only on the input signal and not on its aliased version $X\left(-z_{1},-z_{2}\right)$. However, if we want to solve the problem of achieving perfect reconstruction, a more convenient approach has to be used. Applying the polyphase decomposition, 
the analysis filters can be split into the following two polyphase components:

$$
\begin{aligned}
H_{i}\left(z_{1}, z_{2}\right)= & H_{i 0}\left(z_{1}^{2}, z_{1} z_{2}\right) \\
& +z_{1}^{-1} H_{i 1}\left(z_{1}^{2}, z_{1} z_{2}\right), \\
& i=0,1,
\end{aligned}
$$

and the polyphase filter matrix associated with analysis filter bank is given by

$$
\boldsymbol{H}_{\mathrm{p}}\left(z_{1}, z_{2}\right)=\left[\begin{array}{ll}
H_{00}\left(z_{1}, z_{2}\right) & H_{01}\left(z_{1}, z_{2}\right) \\
H_{10}\left(z_{1}, z_{2}\right) & H_{11}\left(z_{1}, z_{2}\right)
\end{array}\right] .
$$

The two cases of interest are

(1) $\boldsymbol{H}_{\mathrm{p}}\left(z_{1}, z_{2}\right)$ is para-unitary, that is, perfect reconstruction is guaranteed with identical analysis and synthesis filters (within reversal).

(2) $\boldsymbol{H}_{\mathrm{p}}\left(z_{1}, z_{2}\right)$ corresponds to linear phase filters and has a determinant equal to a delay, that is, perfect reconstruction is possible with linear phase FIR filters.

It turns out that, as in the two channel onedimensional case, (1) and (2) are mutually exclusive (except for trivial two-tap filters) [21].

An interesting IIR solution for the para-unitary case was suggested by Ansari in [2] and uses the following choice of filters:

$$
\begin{aligned}
H_{i}\left(z_{1}, z_{2}\right) & =\frac{1}{2}\left(A_{0}\left(z_{1}, z_{2}\right) A_{0}\left(z_{1} z_{2}^{-1}\right)\right. \\
& +(-1)^{i} z_{1}^{-1}\left(A_{1}\left(z_{1} z_{2}\right) A_{1}\left(z_{1} z_{2}^{-1}\right)\right), \quad i=0,1,
\end{aligned}
$$

where $A_{i}(z)$ are one-dimensional allpass functions. One can verify that due to the allpass property of $A_{i}(z)(7)$ is satisfied.

Since in our case one of the dimensions is time we cannot use IIR filters (the reconstruction becomes anti-causal). One approach to design a para-unitary FIR filter bank is to solve (7) numerically under constraints on the filter quality. This leads to a constrained non-linear optimization problem and design examples can be found in
$[1,22]$. A potential problem with this method is that one has no control over the filter coefficients and quantization of the coefficients will result in imperfect reconstruction. It is therefore desirable to find structures that guarantee the perfect reconstruction property regardless of the coefficient quantization.

\section{Cascade structures for perfect reconstruction FIR filter banks}

\subsection{The para-unitary case}

The FIR para-unitary case has some structural properties imposed by ( 7 ) (see Appendix B)

- The filter $H_{1}\left(z_{1}, z_{2}\right)$ is completely specified by the filter $H_{0}\left(z_{1}, z_{2}\right)$ by modulation with $(-1)^{n_{1}+n_{2}}$ and reversal.

- The two polyphase components of $H_{0}\left(z_{1}, z_{2}\right)$ have to be of the same size.

- Finally, the polyphase components of $H_{0}\left(z_{1}, z_{2}\right)$ have to meet

$$
\begin{aligned}
& H_{00}\left(z_{1}, z_{2}\right) H_{00}\left(z_{1}^{-1}, z_{2}^{-1}\right) \\
& \quad+H_{01}\left(z_{1}, z_{2}\right) H_{01}\left(z_{1}^{-1}, z_{2}^{-1}\right)=1 .
\end{aligned}
$$

Equation (14) can now be met through optimizing the coefficients of the first filter and thus specifying all the other ones in the system. Rather then using a numerical approach, we derive cascade structures which produce structurally para-unitary filter banks and thus maintain the perfect reconstruction property regardless of coefficient quantization:

$$
\boldsymbol{H}_{\mathrm{p}}\left(z_{\mathbf{1}}, z_{2}\right)=\boldsymbol{U}_{\boldsymbol{K}-1} \prod_{\boldsymbol{i}=k-2}^{0} \boldsymbol{D}_{i}\left(z_{\mathbf{l}}, z_{2}\right) \boldsymbol{U}_{i},
$$

where $\boldsymbol{D}\left(z_{1}, z_{2}\right)$ is a diagonal matrix of delays and $U_{\mathrm{i}}$ are unitary matrices. It is easy to verify that $\boldsymbol{H}_{\mathrm{p}}\left(z_{1}, z_{2}\right)$ in (15) is para-unitary since all the blocks are para-unitary, but completeness of the structure is not guaranteed, unlike in the onedimensional case [10]. A particular choice of interest for generating (approximately) diamond 
shaped filters is

$$
\begin{aligned}
\boldsymbol{H}_{\mathrm{p}}\left(z_{1}, z_{2}\right) & =\left[\begin{array}{cc}
1 & a_{2 K-1} \\
-a_{2 K-1} & 1
\end{array}\right] \\
& \cdot \prod_{i=K-1}^{0}\left[\begin{array}{cc}
1 & \\
& z_{1}^{-1} z_{2}^{-1}
\end{array}\right]\left[\begin{array}{cc}
1 & a_{2 i+1} \\
-a_{2 i+1} & 1
\end{array}\right] \\
& \cdot\left[\begin{array}{ll}
1 & \\
& z_{1}^{-1} z_{2}
\end{array}\right]\left[\begin{array}{cc}
1 & a_{2 i} \\
-a_{2 i} & 1
\end{array}\right] .
\end{aligned}
$$

The impulse responses of the smallest filter pair obtained with $K=1$ in (16) are hence

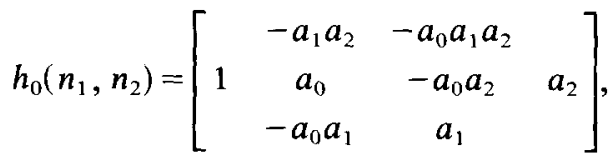

$$
\begin{aligned}
& h_{1}\left(n_{1}, n_{2}\right)=\left[\begin{array}{cccc} 
& -a_{1} & -a_{0} a_{1} & \\
-a_{2} & -a_{0} a_{2} & -a_{0} & 1 \\
& a_{0} a_{1} a_{2} & -a_{1} a_{2} &
\end{array}\right] \text {. }
\end{aligned}
$$

The reconstruction filters are the same (within reversal), except for a scaling factor of $1 / \prod(1+$ $\left.a_{i}^{2}\right)$.

\subsection{The linear phase case}

Obviously, the filters in (17), (18) are never linear phase. Actually, para-unitariness excludes linear phase in the two channel case except for the trivial two-tap filters. For linear phase behavior, we require centro-symmetry, that is,

$$
z_{1}^{-K_{1}} z_{2}^{-K_{2}} H_{i}\left(z_{1}^{-1}, z_{2}^{-1}\right)= \pm H_{i}\left(z_{1}, z_{2}\right) \text {. }
$$

Again, certain constraints have to be met by the filters so as to meet (19) and the perfect reconstruction property. Requiring that the determinant of $\boldsymbol{H}_{\mathrm{p}}\left(z_{1}, z_{2}\right)$ be a pure delay we guarantee that there is a perfect reconstruction FIR solution. Then, if the filters $H_{0}\left(z_{1}, z_{2}\right)$ and $H_{1}\left(z_{1}, z_{2}\right)$ must be of the same size, they must also have polyphase components of the same size (this follows from the symmetry of the two-dimensional polynomials involved). Thus, for example, for a diamond shaped filter

$$
H_{0}\left(z_{1}, z_{2}\right)=1+z_{1}^{-1} z_{2}^{-1}+a z_{1}^{-1}+z_{1} z_{2}+z_{1}^{-2},
$$

one cannot have a same size filter $H_{1}\left(z_{1}, z_{2}\right)$ that achieves perfect reconstruction. However, a different size filter $H_{1}\left(z_{1}, z_{2}\right)$ can be found that satisfies the perfect reconstruction property as will be shown shortly. Also, see Appendix A for a more thorough discussion.

First, let us describe a cascade structure that leads to same size (though not of the desired perfect diamond shape) linear phase filters:

$$
\begin{aligned}
\boldsymbol{H}_{\mathrm{p}}\left(z_{1}, z_{2}\right)= & {\left[\begin{array}{rr}
1 & 1 \\
1 & -1
\end{array} \prod_{i=k}^{0}\left[\begin{array}{ll}
1 & \\
& z_{1}^{-1} z_{2}^{-1}
\end{array}\right]\right.} \\
& \cdot\left[\begin{array}{rr}
1 & a_{2 i+1} \\
a_{2 i+1} & 1
\end{array}\right] \\
& \cdot\left[\begin{array}{cc}
1 & \\
& z_{1}^{-1} z_{2}
\end{array}\right]\left[\begin{array}{cc}
1 & a_{2 i} \\
a_{2 i} & 1
\end{array}\right] .
\end{aligned}
$$

The blocks in the cascade meet the linear phase condition given in [8] and thus the resulting bank is linear phase. The impulse responses of the smallest possible filter pair are given by

$$
\begin{aligned}
& h_{0}\left(n_{1}, n_{2}\right)=\left[\begin{array}{cccc} 
& a_{0} & a_{0} a_{1} & \\
1 & a_{1} & a_{1} & 1 \\
& a_{0} a_{1} & a_{0} &
\end{array}\right], \\
& h_{1}\left(n_{1}, n_{2}\right)=\left[\begin{array}{cccc}
-a_{0} & -a_{0} a_{1} & \\
1 & a_{1} & -a_{1} & -1 \\
& a_{0} a_{1} & a_{0} &
\end{array}\right] .
\end{aligned}
$$

Like para-unitary filters, they only approximate the diamond shape. Note that they have centrosymmetry, but not left-right or top-bottom symmetry. If one desires the perfect diamond shaped filters, as well as top-bottom, left-right symmetry, one has to resort to different size low- and highpass filters. The pair of filters $H_{0}\left(z_{1}, z_{2}\right)$ and $H_{1}\left(z_{1}, z_{2}\right)$ with impulse responses,

$$
\left[\begin{array}{lll} 
& 1 & \\
b & a & b \\
& 1 &
\end{array}\right],
$$

$\left[\begin{array}{ccccc} & & 1 & & \\ & b+c / a & a & b+c / a & \\ b c / a & c & d & c & b c / a \\ & b+c / a & a & b+c / a & \end{array}\right]$, 
leads to perfect reconstruction $[10,20]$. Cascades of the polyphase matrices corresponding to the filters in (23) and (24) will generate larger filters retaining the same symmetry properties, and still allowing perfect FIR reconstruction [10]. By choosing $b=1$ and $c=a$ one obtains additional circular symmetry (the property is also retained when cascading the polyphase matrices). A useful example is obtained with $a=-4$ and $d=-28$ where the impulse responses become (with the reversed sign for the second filter)

$$
\begin{aligned}
& {\left[\begin{array}{rrr} 
& 1 & \\
1 & -4 & 1 \\
& 1 &
\end{array}\right],} \\
& {\left[\begin{array}{rrrrr} 
& & -1 & & \\
& -2 & 4 & -2 & \\
-1 & 4 & 28 & 4 & -1 \\
& -2 & 4 & -2 & \\
& & -1 & &
\end{array}\right] .}
\end{aligned}
$$

The magnitude of the frequency response of (26) is given in Fig. 7(a).

So far, we have given constructions of perfect reconstruction filter banks. One nice property is the easy control one has over the filter coefficients, which is useful in order to derive low complexity filters. Though we know that the cascade structure given is not complete, the first pair of useful filters given by (21), (22) covers the whole space of linear phase $4 \times 3$ filters (except for some permutations). The proof is given in Appendix C.

\section{Applications}

\subsection{A two channel system: interlaced and deinterlacing channel}

Interlaced signals are in wide use because they represent (arguably) a better trade-off between spatio-temporal resolution and bandwidth than non-interlaced signals. A progressive HDTV signal can have a luminance bandwidth as high as $150 \mathrm{MHz}$ and at present this poses technological difficulties for both camera and monitor. In the long range, it appears desirable to evolve toward non-interlaced video signals, in particular for professional applications of HDTV; the perfect reconstruction techniques derived in Section 4 are a good tool to provide a representation of an HDTV signal with both an interlaced channel and a 'deinterlacing' channel.

The two channels (interlaced plus the deinterlacing one) can allow evolution from interlaced to progressive video. In such a system an interlaced receiver would use only the basic interlaced signal, while an advanced receiver would reconstruct a non-interlaced signal with higher spatio-temporal resolution. When using low complexity spatiotemporal filter banks, the amount of processing and the number of field/frame stores is comparable to a more conventional motion adaptive spatial/temporal deinterlacing technique [14], but with potentially better results since the full resolution of the non-interlaced signal could always be recovered. In the perspective of a digital system, the deinterlacing signal could be compressed because of its relatively low entropy and the masking properties of the human visual system.

\subsection{Interlaced video with progressive subchannel}

It is generally admitted that given the same number of samples non-interlaced video signals are easier to code than interlaced signals, the primary reason being that the vertical correlation in a field (from interlaced) is smaller than the vertical correlation in a non-interlaced frame. Indeed, a field extracted from an interlaced video signal contains a significant amount of vertical aliasing. Also when using interframe prediction with interlaced video, the predictor should be able to switch between previous frame prediction and previous field prediction. For both of these reasons, it appears useful to split the interlaced video into a progressive reduced frame rate subchannel and a signal that contains only high vertical and high temporal frequency information. If the energy contained in the high vertical and high temporal frequency band 

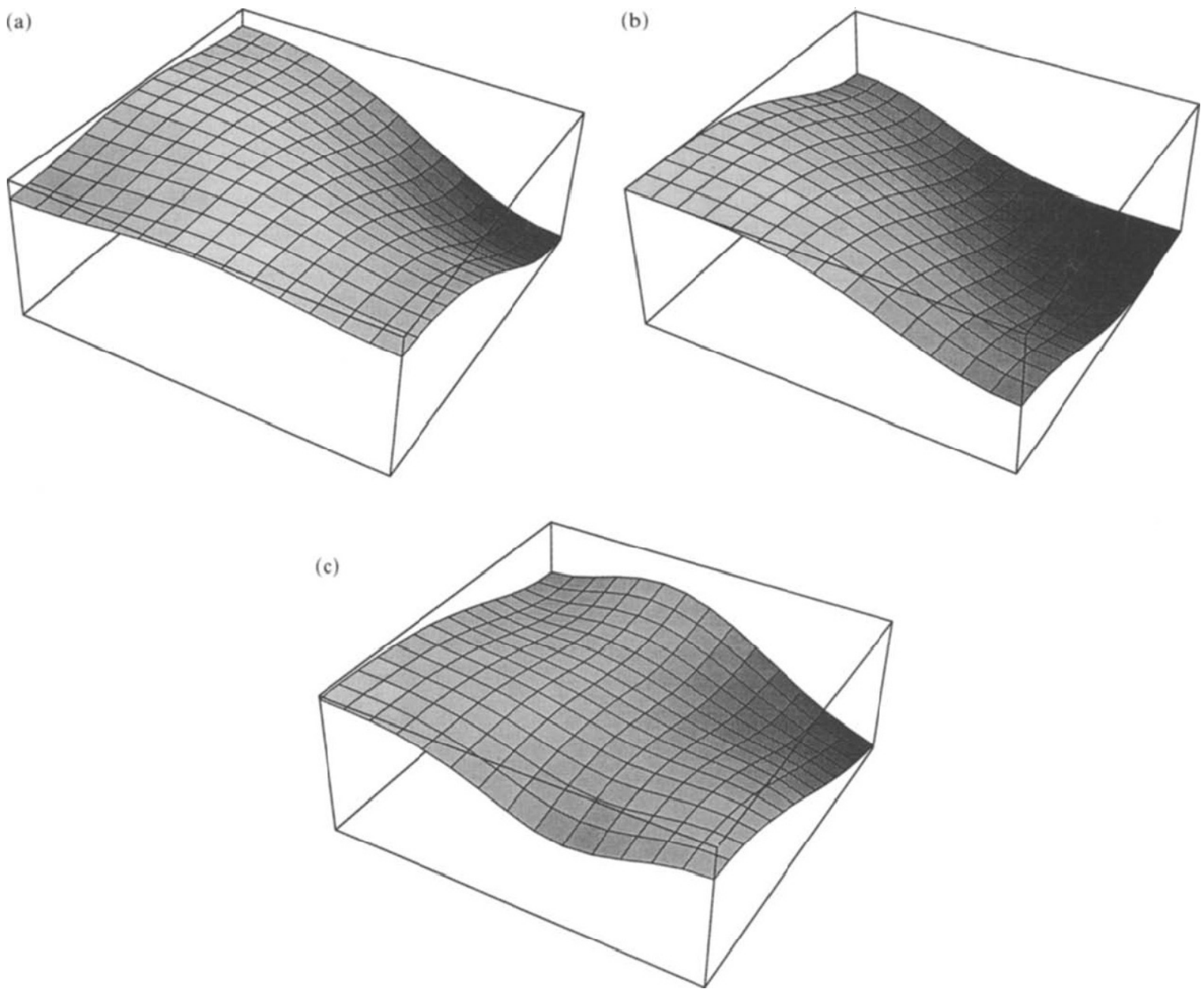

Fig. 7. Frequency responses of (a) linear phase $5 \times 5$ filter given by (26), (b) reference $7 \times 7$ linear phase filter given by (29), (c) para-unitary filter given by (17).

is low, coding those two channels independently can be very efficient.

\section{Implementation and results}

The implementation of the proposed scheme is shown in Fig. 3. The progressive sequence used $(256 \times 256,40$ frames) was generated by diagonally panning the 'Kiel Harbor' image in 1-pixel wide steps.

Using the structures given in Section 4, three sets of filters were designed corresponding to both the para-unitary and the linear phase case. These filters were used with the quincunx sampling matrix in the two steps of the subband scheme in Fig. 3. The para-unitary set is obtained by substituting $a_{0}=2, a_{1}=0.5$ and $a_{2}=1$ in (17), (18) and the first linear phase set substituting the same coefficients into (21), (22). Due to the fact that both filter pairs are generated with cascade structures producing polyphase components of the same size, they only approximate the desired perfect diamond shape. The third set designed solves this problem since it consists of the lowpass filter of the form (26) and the highpass filter of the form 
(25). Note that the filters obtained are just initial designs and that more work has to be done in optimizing the coefficients so as to achieve the desired frequency response. Note that these filters have very low complexity. As a reference a very good $7 \times 7$ diamond shaped lowpass filter was designed from a one-dimensional filter with the following $z$-transform:

$$
\begin{aligned}
H(z) & =-1+9 z^{-2}+16 z^{-3}+9 z^{-4}-z^{-6} \\
& =H_{0}\left(z^{2}\right)+z^{-1} H_{1}\left(z^{2}\right) .
\end{aligned}
$$

The two-dimensional filter is then obtained as [2]

$$
\begin{aligned}
D\left(z_{1}, z_{2}\right)= & H_{0}\left(z_{1} z_{2}\right) H_{0}\left(z_{1} z_{2}^{-1}\right) \\
& +z_{1}^{-1} H_{1}\left(z_{1} z_{2}\right) H_{1}\left(z_{1} z_{2}^{-1}\right),
\end{aligned}
$$

producing the following impulse response:

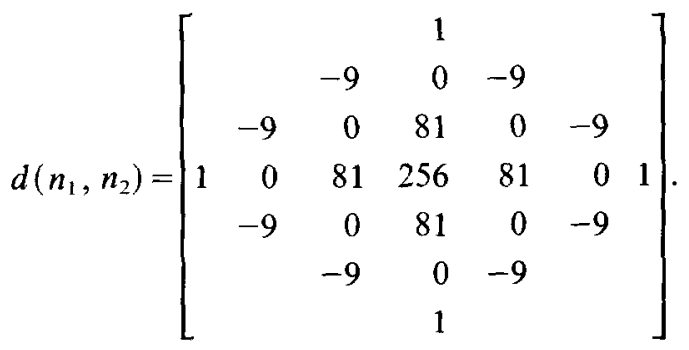

Its frequency response is given in Fig. 7(b).

As the indication of possible compression the bit-rates of PCM and run-length encoded high bands were computed in both the para-unitary and the linear phase case. The quality and bit rate of the reconstructed sequence are thus controlled by adjusting the step of the uniform quantizer and its 'dead zone' [6]. Some results are shown in Table 1 , where the 'dead zone' used was twice the width of the quantization step. Comparing the frequency responses of the lowpass filters from the linear phase diamond shape set, the para-unitary set and the reference one, it can be seen that the reference one achieves very good out-of-band rejection, but it has rather sharp response, and the para-unitary one has very poor out-of-band rejection. A good compromise between the two is the diamond shaped one which has smooth frequency response and decent out-of-band rejection. Thus the recon-
Table 1

Bit-rates of the high band in Fig. 1 and mean square error. MSE is computed for the case where just the high channel is coded

\begin{tabular}{llll}
$\begin{array}{l}\text { Number of } \\
\text { quantization levels }\end{array}$ & bit rate [bits/pel] & MSE \\
\hline $31 \quad$ Linear phase & 1.315574 & 3.61488 \\
& para-unitary & 1.576009 & 2.859315 \\
$15 \quad$ linear phase & 1.041300 & 6.726135 \\
& para-unitary & 1.135823 & 5.75076
\end{tabular}

structed sequences are visually more pleasing when using either set of linear phase filters ((21), (22) with coefficients as above or $(26,25))$ compared to the para-unitary one for the same bit rates.

Using the diamond shaped linear phase set (26), (25) also produced a visually better interlaced sequence as opposed to the one when the reference filter given by (29) was used.

The result after the two steps of subband splitting (as in Fig. 3) with representative frames from the sequence for the (26), (25) set is shown in Fig. $8(\mathrm{a})$. Figure $8(\mathrm{~b}, \mathrm{c}, \mathrm{d})$ show a representative frame from interlaced sequences obtained by using the reference linear phase filter and the lowpass filters (21), (26).

Note that all the results were observed on a digital real-time video display.

\section{Conclusion and further work}

This paper has demonstrated how to go from progressive to interlaced scanning in a way that allows perfect reconstruction of the original progressive sequence. It can also be used to go from interlaced to progressive scanning and back. Perfect reconstruction filter banks were derived, cascade structures that achieve very low complexity as well as linear phase were shown. The initial results on sequences show that this could be an attractive way to derive compatible channels as well as to produce a decomposition useful for coding. 


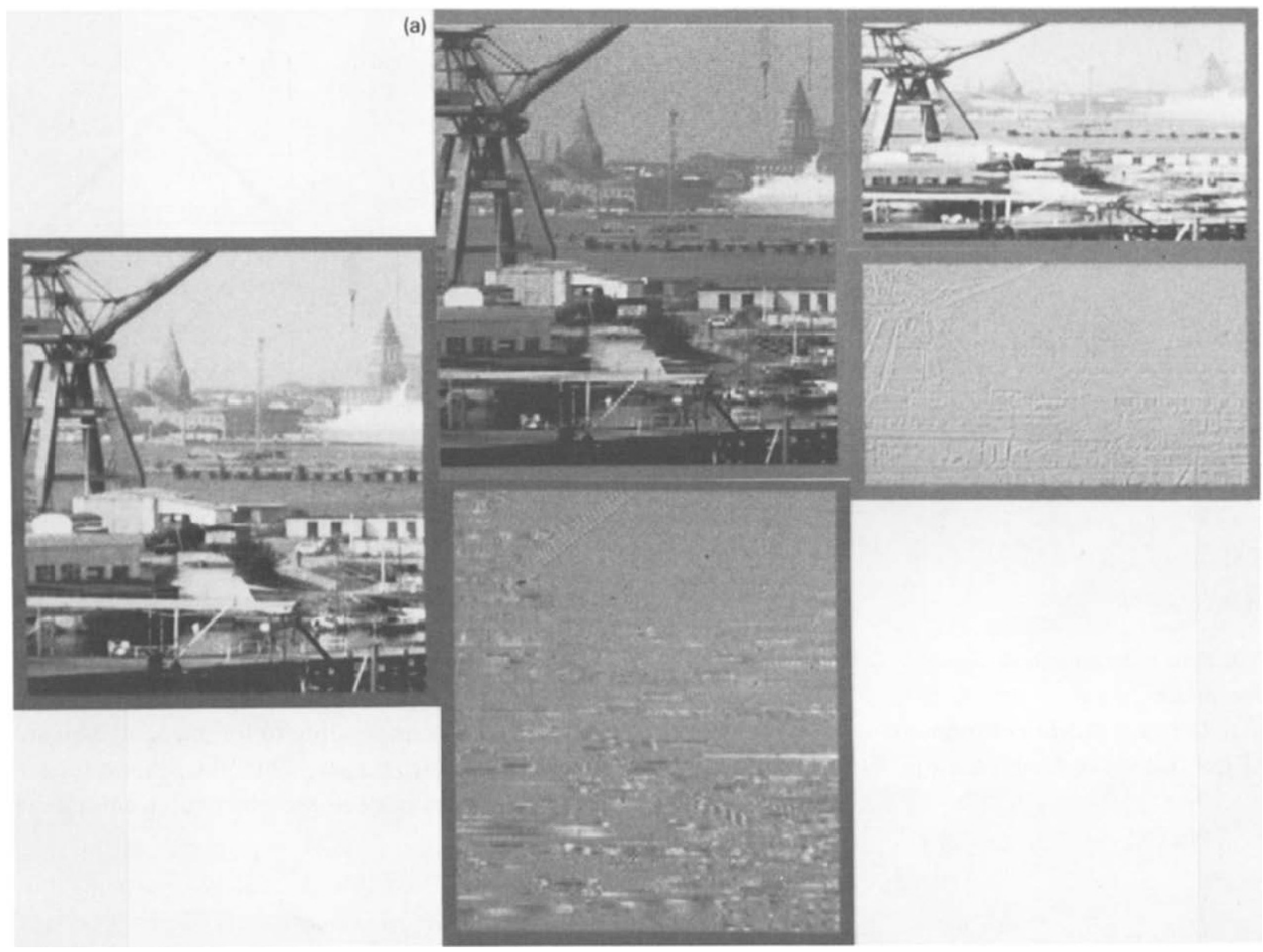

Fig. 7 -continued

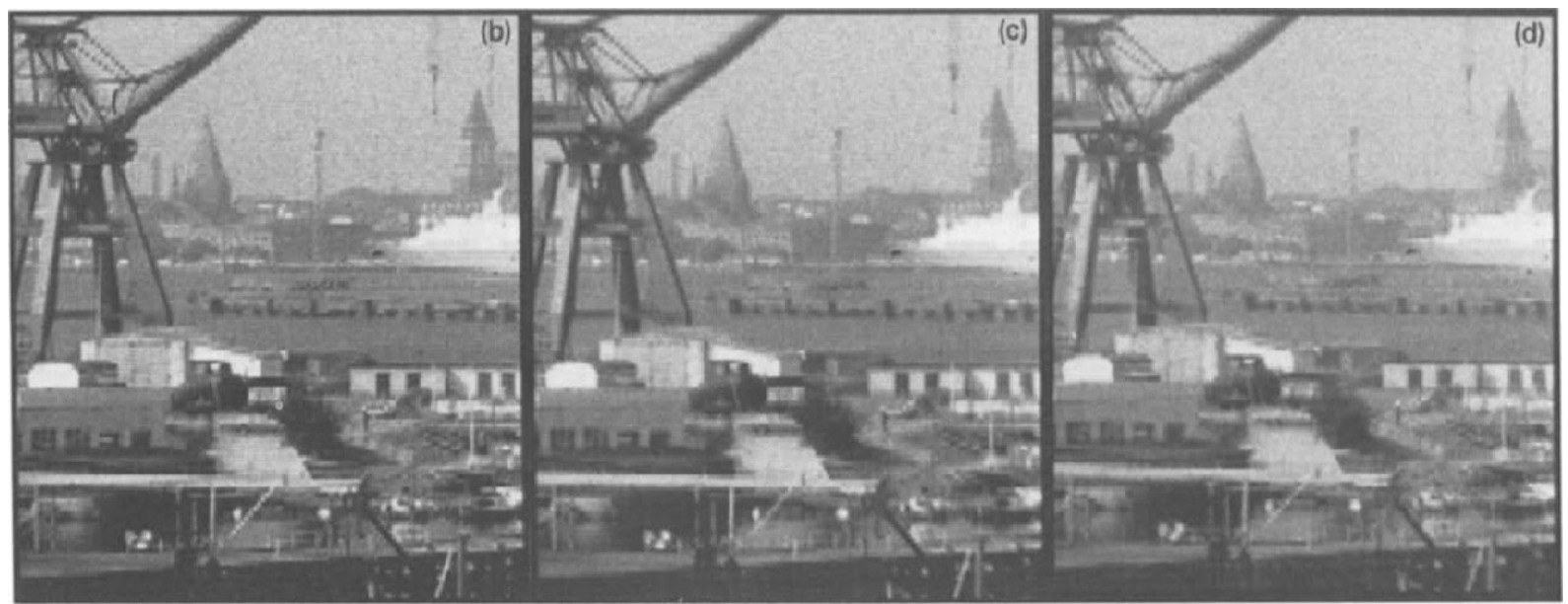

Fig. 8. Results from a pan of Kiel Harbor, (a) after two step splitting as described in Fig. 3, (b) frame from the interlaced sequence obtained with filter $(26)$, (c) frame from the interlaced sequence obtained with filter (29), (d) frame from the interlaced sequence obtained with filter (17). 


\section{Appendix A. Linear phase solution}

Let us first introduce some notation. A twodimensional 'persymmetric' polynomial $p(x, y)$ is the one for which $p(x, y)=x^{n} y^{m} p\left(x^{-1}, y^{-1}\right)$. A two-dimensional 'antipersymmetric' polynomial $p(x, y)$ is the one for which $p(x, y)=$ $-x^{n} y^{m} p\left(x^{-1}, y^{-1}\right)$. The product of two persymmetric polynomials is again persymmetric, while the sum of two persymmetric polynomials of the same size is again persymmetric. Also, in this section we will use the following form of the quincunx sampling matrix:

$$
D=\left[\begin{array}{rr}
1 & 1 \\
-1 & 1
\end{array}\right]
$$

\section{A.1. Case 1}

Let us consider first the case where both filters are made causal in one dimension in such a way that the polyphase components of the filters are of the following form (see Fig. 9):

$$
\begin{aligned}
& H_{00}\left(z_{1}, z_{2}\right)=\sum_{i=0}^{k} \sum_{j=0}^{k} a_{i j} z_{1}^{i} z_{2}^{j}, \\
& H_{01}\left(z_{1}, z_{2}\right)=\sum_{i=0}^{k-1} \sum_{j=0}^{k-1} b_{i j} z_{1}^{i} z_{2}^{j}, \\
& H_{10}\left(z_{1}, z_{2}\right)=\sum_{i=0}^{l} \sum_{j=0}^{l} c_{i j} z_{1}^{i} z_{2}^{j}, \\
& H_{11}\left(z_{1}, z_{2}\right)=\sum_{i=0}^{l-1} \sum_{j=0}^{l-1} d_{i j} z_{1}^{i} z_{2}^{j} .
\end{aligned}
$$

Since the filters are supposed to be linear phase and diamond shaped at the same time, all four polyphase components are persymmetric. The determinant of the corresponding polyphase matrix $\boldsymbol{H}_{\mathrm{p}}\left(z_{1}, z_{2}\right)$ is then

$$
\begin{aligned}
D\left(z_{1}, z_{2}\right)= & H_{00}\left(z_{1}, z_{2}\right) H_{11}\left(z_{1}, z_{2}\right) \\
& -H_{01}\left(z_{1}, z_{2}\right) H_{10}\left(z_{1}, z_{2}\right) \\
= & p\left(z_{1}, z_{2}\right)-q\left(z_{1}, z_{2}\right) .
\end{aligned}
$$

Since all four involved polynomials are persymmetric $p\left(z_{1}, z_{2}\right), q\left(z_{1}, z_{2}\right)$ are persymmetric as well.
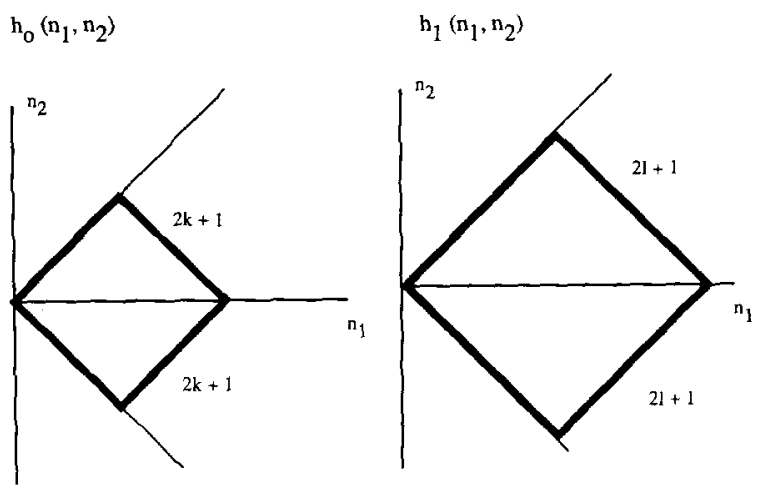

Fig. 9. Perfectly diamond shaped filter pair. Diamond shaped filter becomes rectangular on the quincunx lattice when matrix $D_{2}$ is used.

\section{A.1.1. Same size filters}

Now $\operatorname{Deg}\left(H_{00}\right)=\operatorname{Deg}\left(H_{\mathrm{t} 0}\right)=(k, k)$, while $\operatorname{Deg}\left(H_{01}\right)=\operatorname{Deg}\left(H_{11}\right)=(k-1, k-1)$. This in turn means that $\operatorname{Deg}(p)=\operatorname{Deg}(q)=(2 k-1,2 k-1)$. The determinant is then a persymmetric polynomial of even length in both directions, which means that it is impossible to force one coefficient to be different from zero, while the other ones are zero, and thus perfect reconstruction cannot be achieved.

\section{A.1.2. Different size filters}

In this case $\operatorname{Deg}\left(H_{00}\right)=(k, k), \operatorname{Deg}\left(H_{01}\right)=$ $(k-1, k-1), \quad \operatorname{Deg}\left(H_{10}\right)=(l, l)$ and $\operatorname{deg}\left(H_{11}\right)=$ $(l-1, l-1)$, which yields $\operatorname{Deg}(p)=\operatorname{Deg}(q)=$ $(k+l-1, k+l-1)$. The determinant thus becomes persymmetric. To obtain a monomial and achieve perfect reconstruction, a necessary condition is that $D\left(z_{1}, z_{2}\right)$ be of odd size in both directions, i.e., that $k$ is even and $l$ odd or vice versa. An example of the solution belonging to this class is given in (23), (24).

\section{A.2. Case 2}

Next we consider what happens if we keep the first filter as it was and displace the second one by $z_{1}$, which reverses the order of the entries in the second row of the polyphase matrix and thus covers all possibilities of linear phase, diamond shaped filters. The polyphase components of the 
second filter thus become

$$
\begin{aligned}
& H_{10}\left(z_{1}, z_{2}\right)=z_{1} z_{2} \sum_{i=0}^{l-1} \sum_{j=0}^{l-1} c_{i j} z_{1}^{i} z_{2}^{j}, \\
& H_{11}\left(z_{1}, z_{2}\right)=\sum_{i=0}^{l} \sum_{j=0}^{l} d_{i j} z_{1}^{i} z_{2}^{j} .
\end{aligned}
$$

Obviously $p$ and $q$ are again persymmetric. As before let us consider two cases.

\section{A.2.1. Same size filters}

Now $\operatorname{Deg}(p)=(2 k, 2 k)$ and $\operatorname{Deg}(q)=$ $(2 k-1,2 k-1)$. Though $p$ and $q$ are not of the same size the determinant is still going to be persymmetric since $q$ is actually of size $(2 k-2) \times$ $(2 k-2)$ but displaced by one along the diagonal. The size of the determinant is now odd in both directions which leads us to assume that the solution might be possible. However the corner coefficients, like for example $a_{00} d_{00}$, have to be zero which ruins the perfect diamond shape of the filters.

\section{A.2.2. Different size filters}

Using the same reasoning as before we can conclude that in case of $k$ even and $l$ odd or vice versa a solution might be possible at the cost of ruining the perfect shape of the filters.

To summarize it is possible to obtain perfect reconstruction diamond shaped filter pair when both filters are made causal in one dimension and when their sizes are $(2 k+1) \times(2 k+1)$ and $(2 l+$ $1) \times(2 l+1)$, where $k$ and $l$ are not both odd or both even at the same time.

\section{Appendix B. Para-unitary solution}

Let us denote $H_{i j_{+}}=H_{i j}\left(z_{1}, z_{2}\right)$ and $H_{i j_{-}}=$ $H_{i j}\left(z_{1}^{-1}, z_{2}^{-1}\right)$. We then obtain

$$
\begin{aligned}
\boldsymbol{H}_{\mathrm{p}}^{\mathrm{T}}\left(z_{1}^{-1}, z_{2}^{-1}\right) \cdot \boldsymbol{H}_{\mathrm{p}}\left(z_{1}, z_{2}\right) \\
=\left[\begin{array}{ll}
H_{00_{+}} H_{00_{-}}+H_{10_{+}} H_{10_{-}} & H_{00_{-}} H_{01_{+}}+H_{10_{-}} H_{11_{+}} \\
H_{01_{-}} H_{00_{+}}+H_{11_{-}} H_{10_{+}} & H_{0_{-}} H_{0_{1_{+}}}+H_{11_{-}} H_{11_{+}}
\end{array}\right] .
\end{aligned}
$$

If we want $\boldsymbol{H}_{\mathrm{p}}$ to be para-unitary, the above must be equal to $I$, from where we can obtain the following equalities:

$$
\begin{aligned}
& H_{00_{+}} H_{00_{-}}+H_{10_{+}} H_{10_{-}}=1 . \\
& H_{00_{-}} H_{01_{+}}+H_{10_{-}} H_{11_{+}}=0, \\
& H_{01_{-}} H_{00_{+}}+H_{11_{-}} H_{10_{+}}=0, \\
& H_{01_{-}} H_{01_{+}}+H_{11_{-}} H_{11_{+}}=1 .
\end{aligned}
$$

Using the same approach as in [17] we can conclude from (39) that $H_{00}$ and $H_{10}$ do not have common polynomial factors since the right-hand side is a non-zero constant. Similarly, from (42) it follows that $H_{01}$ and $H_{11}$ do not have common factors. Bearing this in mind (40) yields that all the polynomial factors of $H_{11}$ are contained in $H_{00}$ and all the polynomial factors of $H_{10}$ are contained in $H_{01}$, that is,

$$
H_{01_{+}}=c_{1} z_{1}^{-k} z_{2}^{-1} H_{10_{-}}, \quad H_{11_{+}}=c_{2} z_{1}^{-m} z_{2}^{-n} H_{00_{-}} .
$$

Substituting this into (40) we conclude that $c_{1}=$ $-c_{2}$ and that $k=m, l=n$. Also from (42) we can see that $\left|c_{1}\right|=1$. Finally, the complete specification of the para-unitary system is given in the following form:

$$
\begin{aligned}
& H_{01_{+}}=z_{1}^{-k} z_{2}^{-1} H_{10_{-},} \\
& H_{11_{+}}=-z_{1}^{-k} z_{2}^{-1} H_{00_{-}} \\
& H_{00_{+}} H_{00_{-}}+H_{10_{+}} H_{10_{-}}=1,
\end{aligned}
$$

from where we can see that the two polyphase components have to be of the same size, which automatically excludes the possibility of having filters of the desired perfect diamond shape. At the same time the previous set of equalities shows that the filter $H_{1}\left(z_{1}, z_{2}\right)$ is completely specified by modulating and reversing filter $H_{0}\left(z_{1}, z_{2}\right)$.

\section{Appendix C. Completeness of the $4 \times 3$ linear phase solution}

In this appendix we show that the linear phase filters given by (21), (22) cover all possible sol- 
utions for the filters with linear phase and of that size. Let the impulse responses of two general $4 \times 3$ linear phase filters be

$$
\begin{aligned}
& h_{0}\left(n_{1}, n_{2}\right)=\left[\begin{array}{llll} 
& a_{0} & b_{0} & \\
c_{0} & d_{0} & d_{0} & c_{0} \\
& b_{0} & a_{0} &
\end{array}\right], \\
& h_{1}\left(n_{1}, n_{2}\right)=\left[\begin{array}{llll} 
& a_{1} & b_{1} & \\
c_{1} & d_{1} & d_{1} & c_{1} \\
& b_{1} & a_{1} &
\end{array}\right] .
\end{aligned}
$$

Computing the determinant of the corresponding polyphase matrix and equating all the coefficients but one to zero, the following set of conditions (in matrix form) is obtained:

$$
\left[\begin{array}{cccc}
b_{0} & -a_{0} & 0 & 0 \\
c_{0} & -d_{0} & a_{0} & -b_{0} \\
d_{0} & -c_{0} & b_{0} & -a_{0} \\
0 & 0 & d_{0} & -c_{0}
\end{array}\right]\left[\begin{array}{l}
a_{1} \\
b_{1} \\
c_{1} \\
d_{1}
\end{array}\right]=\left[\begin{array}{l}
0 \\
0 \\
0 \\
0
\end{array}\right],
$$

which shows that we are solving for the coefficients of the second filter given the coefficients of the first one. Equation (49) has a nontrivial solution iff the determinant of the above matrix equals zero. This in turn leads to the following (with $c_{0}=1$ which is just scaling):

$$
-a_{0} b_{0}+a_{0}^{2} d_{0}+b_{0}^{2} d_{0}-a_{0} b_{0} d_{0}^{2}=0 .
$$

Solving for $b_{0}$ we obtain

$$
b_{01}=\frac{a_{0}}{d_{0}}, \quad b_{02}=a_{0} d_{0} .
$$

Taking the second solution for example, the coefficients of the second filter become

$$
a_{1}=-\frac{a_{0}}{d_{0}}, \quad b_{1}=-a_{0}, \quad c_{1}=\frac{1}{d_{0}}, \quad d_{1}=1 .
$$

Multiplying (52) by $d_{0}$ (scaling), we obtain the following impulse responses for the general linear phase perfect reconstruction pair:

$$
h_{0}\left(n_{1}, n_{2}\right)=\left[\begin{array}{cccc} 
& a_{0} & a_{0} d_{0} & \\
1 & d_{0} & d_{0} & 1 \\
& a_{0} d_{0} & a_{0} &
\end{array}\right],
$$

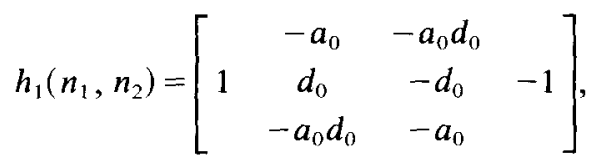

which is exactly how (21), (22) look like. The only thing that is left to check is whether the coefficients that are forbidden in the cascade structure, that is, the ones which would produce singular blocks, are allowed in the general solution. Let us look at the only non-zero coefficient in the determinant of the polyphase matrix:

$$
a_{0} a_{1}-b_{0} b_{1}+c_{0} c_{1}-d_{0} d_{1} \neq 0 .
$$

Substituting the solutions for the coefficients obtained earlier into (55) yields

$$
-a_{0}^{2}+a_{0}^{2} d_{0}^{2}+1-d_{0}^{2}=\left(1-a_{0}^{2}\right)\left(1-d_{0}^{2}\right) \neq 0,
$$

resulting in $\left|a_{0}\right| \neq 1$ and $\left|d_{0}\right| \neq 1$ which are exactly the values not allowed in the cascade structure. The solution corresponding to $b_{01}$ is obtained by interchanging the order of diagonal matrices in (20) which concludes the proof. Let us note that for the larger sizes the above approach becomes quite cumbersome and thus the question of how complete the larger cases are has yet to be answeed.

\section{References}

[1] E. Adelson and E. Simoncelli, "Orthogonal pyramid transforms for image coding", in: Proc. SPIE Conf. on Visual Communications and Image Processing, 1987, pp. 50-58.

[2] R. Ansari, "Two dimensional IIR filters for exact reconstruction in tree-structured subband decomposition", Electron. Letters, Vol. 23, June 1987, pp. 633-634.

[3] R. Ansari, H. Gaggioni and D. LeGall, "HDTV coding using a non-rectangular subband decomposition", in: Proc. SPIE Conf. on Visual Communications and Image Processing, Cambridge, MA, November 1988, pp. 821-824.

[4] J. Cassels, An Introduction to the Geometry of Numbers, Springer, Berlin, 1971

[5] E. Dubois, "The sampling and reconstruction of timevarying imagery with application in video systems", Proc. IEEE, Vol. 73, April 1985, pp. 502-522.

[6] H. Gharavi and A. Tabatabai, "Sub-band coding of monochrome and color images", IEEE Trans. Circuits and Systems, Vol. 35, February 1988, pp. 207-214. 
[7] M. Isnardi, J. Fuhrer, T. Smith, J. Koslov, B. Roeder and W. Wedam, "Encoding for compatibility and recoverability in the ACTV system", IEEE Trans. Broadcasting, Vol. 33, December 1987, pp. 116-123.

[8] G. Karlsson and M. Vetterli, "Theory of two-dimensional multirate filter banks", IEEE Trans. Acoust. Speech Signal Process, Vol. 38, June 1990, pp. 925-937.

[9] G. Karlsson and M. Vetterli, "Three dimensional subband coding of video", in: Proc. IEEE Internat. Conf. Acoust. Speech Signal Process., April 1988, 1100-1103.

[10] J. Kovačević, M. Vetterli and G. Karlsson, "Design of multidimensional filter banks for non-separable sampling", in: Proc. IEEE Internat. Symp. Circuits and Systems, New Orleans, LA, May 1990, pp. 2004-2008.

[11] D.J. LeGall, H. Gaggioni and C.T. Chen, "Transmission of HDTV signals under $140 \mathrm{Mbit} / \mathrm{s}$ using a sub-band decomposition and discrete cosine transform coding", in: L. Chiariglione, ed., Signal Processing of HDTV, NorthHolland, Amsterdam, 1988, pp. 287-293.

[12] W. F. Schreiber et al., "Channel compatible 6-MHz HDTV distribution systems", Tech, Rep. 79, MIT, January 1988.

[13] E. Simoncelli and E. Adelson, "Non-separable extensions of quadrature mirror filters to multiple dimensions", in Proc. IEEE, April 1990.

[14] G. Tonge, "Signal processing for higher definition television", IBA Tech. Rev., Vol. 21, November 1983, pp. 13-26.

[15] M. Tsinberg, "ENTSC two-channel compatible HDTV system", IEEE Trans. Cons. Electronics, Vol. 33, August 1987, pp. 146-153.
[16] P.P. Vaidyanathan, "Quadrature mirror filter banks, $M$ band extensions and perfect reconstruction technique", IEEE Acoust. Speech Signal Process. Mag., Vol. 4, July 1987, pp. 4-20.

[17] P.P. Vaidyanathan and Z. Doğanata, "The role of lossless systems in modern digital signal processing: A tutorial", IEEE Trans. Education, Vol. 32, August 1989, pp. 181-197.

[18] M. Vetterli, "Multi-dimensional sub-band coding: some theory and algorithms", Signal Processing, Vol. 6, No. 2, February 1984, pp. 97-112.

[19] M. Vetterli, "Multirate filter banks for subband coding", in: J. W. Woods, ed., Subband Coding of Images, Kluwer Academic Publishers, Dordrecht, 1990, to appear.

[20] M. Vetterli, J. Kovačević and D. LeGall, "Perfect reconstruction filter banks for HDTV representation and coding", in: Proc. of the Third Internat. Workshop on HDTV, Torino, Italy, September 1989.

[21] M. Vetterli and D. LeGall, "Perfect reconstruction FIR filter banks: some properties and factorizations", IEEE Trans. Acoust. Speech Signal Process., Vol. 37, July 1989, pp. 1057-1071.

[22] E. Viscito and J. Allebach, "The analysis and design of multidimensional FIR perfect reconstruction filter banks for arbitrary sampling lattices", submitted.

[23] F. M. Wang, D. Anastassiou and A.M. Netravali, "Motion compensated deinterlacing of video sequences", in: Proc. of the Third Internat. Workshop on HDTV, Torino, Italy, September 1989.

[24] J.W. Woods and S.D. O'Neil, "Sub-band coding of images", IEEE Trans Acoust. Speech Signal Process,, Vol. 34, October 1986, pp. 1278-1288. 